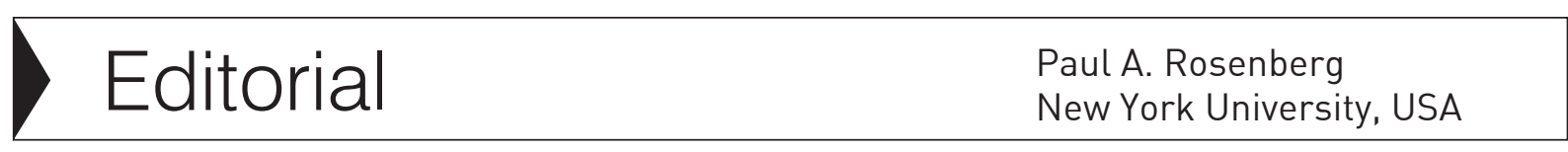

\section{Treatment Planning: A New Paradigm}

The introduction and success of dental implants has had positive results for many patients but has also caused turmoil in the treatment planning arena. The advent of implants as a predictable clinical treatment modality has modified the traditional endodontic treatment planning paradigm. Previously, patients having a tooth at risk were evaluated by a generalist or sent to an endodontist to determine the prognosis of an endodontic procedure. Today, that pathway has been markedly altered. Patients are commonly sent to periodontists or oral surgeons to determine the best treatment plan for the patient. It is not surprising that those specialists' skill set and experience often leads them to conclude that an implant is the best plan. The endodontist is often shut out of the treatment planning process. Many endodontists in the U.S. believe that teeth that could be saved by basic endodontic procedures are no longer even considered for treatment.

In an environment colored by overstatements and half- truths, facts can be difficult to find. Efforts have been made to compare the prognosis of an implant to that of an endodontically treated tooth. Anecdotal evidence is often seized upon to prove a debating point. However, studies with small sample sizes, varied recall periods and different outcomes measures cannot be compared. A comparison between the prognosis of an endodontically treated tooth and an implant is dependent on many variables. Comparisons between the clinical modalities are complicated by confounding variables. It has been said that in clinical research there are variables that we can recognize and control, other variables that we can recognize but can not control and still others that we neither recognize nor control.

The best available evidence indicates that when appropriately completed, both endodontics and implants have a remarkably high level of success. However, there are factors that modify the prognosis in both situations. The systemic health of the patient, smoking, periodontal problems as well as tooth anatomy and occlusion may all affect the outcome of the case. What does seem clear is that the patient is best served by retaining their natural dentition as long as the prognosis for long- term retention is positive. It makes no sense to extract a treatable tooth if a highly predictable endodontic procedure can be performed. On the other hand, it makes no sense for a patient to invest in root canal therapy, a post and crown if the prognosis is highly questionable and an implant with a good prognosis can be placed.

Unfortunately, the endodontist is often marginalized in today's treatment planning process. This is to the detriment of the patient. A central question concerning this matter is, "How are our patients best served?" A patient would benefit from the participation of an endodontist in the development of their treatment plan. In the current environment this will require an endodontist with additional knowledge.

An endodontist with "dual training" that is endodontic and implant training would represent a formidable practitioner. The patient would benefit by seeing a clinician with skills in both areas, thus avoiding a bias in treatment planning. All that separates the endodontist from that role is the necessary education in implantology. In the U.S., some post-graduate programs have already taken steps in that direction. At least one program is building a faculty with dual training and some of the faculty are already including implant placement in their practices. Other post-graduate curriculums are including aspects of implant dentistry and are encouraging their students to go further with this phase of their education. Consider the value to the patient of seeing a clinician who can examine them from the perspectives of endodontics and implants and determine their best course of treatment without a bias.

Specialties have always redefined themselves. Evidence of change abounds. Simply consider the periodontist of today with their emphasis on implants as compared to the periodontist of twenty years ago and their focus on tooth retention. The question is not who should be doing implants. The appropriate question is how we can best serve our patients. It is time for endodontists to redefine our specialty in order to provide our patients with the highest level of care. 